\title{
Testing modified gravity at large distances with the HI Nearby Galaxy Survey's rotation curves
}

\author{
Jorge Mastache \\ Departamento de Física Teórica, Instituto de Física, \\ Universidad Nacional Autónoma de México, México D.F., 04510, México. \\ Jorge L. Cervantes-Cota* \\ Departamento de Física, Instituto Nacional de Investigaciones Nucleares, \\ Apartado Postal 18-1027, Col. Escandón, México DF,11801, México. \\ Axel de la Macorra \\ Departamento de Física Teórica, Instituto de Física, \\ Universidad Nacional Autónoma de México, México D.F., 04510, México.
}

\begin{abstract}
Recently a new -quantum motivated- theory of gravity has been proposed that modifies the standard Newtonian potential at large distances when spherical symmetry is considered. Accordingly, Newtonian gravity is altered by adding an extra Rindler acceleration term that has to be phenomenologically determined. Here we consider a standard and a powerlaw generalization of the Rindler modified Newtonian potential. The new terms in the gravitational potential are hypothesized to play the role of dark matter in galaxies. Our galactic model includes the mass of the integrated gas, and stars for which we consider three stellar mass functions (Kroupa, diet-Salpeter, and free mass model). We test this idea by fitting rotation curves of seventeen low surface brightness galaxies from The HI Nearby Galaxy Survey (THINGS). We find that the Rindler parameters do not perform a suitable fit to the rotation curves in comparison to standard dark matter profiles (Navarro-Frenk-White and Burkert) and, in addition, the computed parameters of the Rindler gravity show a high spread, posing the model as a nonacceptable alternative to dark matter.
\end{abstract}

PACS numbers: 98.62.Dm, 95.30.Cq, 98.62.Gq

\footnotetext{
* jorge.cervantes@inin.gob.mx
} 


\section{INTRODUCTION}

The standard model of cosmology needs large amounts of dark matter and dark energy to fit data of several cosmological and astrophysical probes, see e.g. Refs. [1, 2]. However, up to date there is no direct evidence that the energy density of dark matter or dark energy is given by particles, and dark energy is difficult to accommodate within the present understanding of quantum field theory. Therefore, alternatives to both dark matter and dark energy are abundant in the literature, see Ref. [3] for current approaches.

Recently a new -quantum motivated- theory of gravity has been proposed that modifies the standard Newtonian potential at large distances when spherical symmetry is considered [4]. The formalism incorporates a Rindler acceleration term that could serve to explain the galactic dynamics without the presence of a dark matter halo [5]. This seems plausible since a rough estimate of the Rindler acceleration is of the order $\sim 10^{-9} \mathrm{~cm} / \mathrm{s}^{2}$, a value similar to MOND's acceleration that has been proven to successfully explain rotation curves without a dark matter halo [6] [8]; other applications to this model have been computed elsewhere [9 13]. The aim of the present work is to test this idea when applied to rotation curves of spirals. To perform this task, we use the HI Nearby Galaxy Survey (THINGS), which collects high spectral resolution data revealing extended measurements of gas rotation velocities and circular baryonic matter trajectories [14]. Given these properties it is adequate to test the new gravity model with THINGS, which has been used to test different core/cusp mass profiles. For disk-dominated galaxies the core and cusp profiles fit equally well, however for low surface brightness galaxies there is a clear preference for core profiles over the cuspy models [15]. Analyses of different high resolution datasets have confirmed this tendency

in past recent years [16 20]. In this work, we present a study of the rotation curves using the new gravity model, taking into account the contribution from gas and the three stellar disk models (Kroupa, diet-Salpeter, free mass). By fitting the models to the data we find that although the fits are achievable for the considered galaxies, in many cases they show high $\chi_{\text {red }}^{2}$ values, and a high spread in the Rindler parameters $(a, n)$. Furthermore, the standard dark matter profiles [Navarro-Frenk-White (NFW) and Burkert] do a better job in the fittings to rotation curves. A very recent work 21] considers the same problem and it uses the same eight (of our seventeen) galaxies for the standard Rindler model $(n=1)$, concluding that for six galaxies their results tend to converge to a single Rindler acceleration parameter. In our case, we observe this evidence too but when one takes into account more galaxies or other stellar galactic models their conclusions do not hold.

This work is organized as follows: In Sec. II we review the basic concepts of galactic dynamics when applied to the Rindler acceleration as implied from Ref. [4]. In Sec. III we describe the THINGS galactic sample and in Sec. IV explain how the gas is treated and the stellar mass models considered, implying different galactic models. Then in Sec. $\mathrm{V}$ we analyze the results yielded from the minimization procedure as best fits of the different models. Finally, the conclusions are drawn in Sec. VI. 


\section{MODIFIED GRAVITY AT LARGE DISTANCES}

In Grumiller's approach [5] the effective potential of a point mass $\left(M_{i}\right)$, without angular momentum contribution, is:

$$
\phi_{i}\left(x_{i}\right)=-G \frac{M_{i}}{x_{i}}+a x_{i}
$$

where $x_{i}=\left|\overrightarrow{x_{i}}\right|=\left|\vec{r}-\vec{r}_{i}^{\prime}\right|, \vec{r}$ is an arbitrary point, $r^{\prime}$ are the particle coordinates, and $a$ is a universal constant, the Rindler acceleration.

For a smooth matter distribution with spherical symmetry, one has

$$
\phi(r)=-G \int \frac{\rho(r \prime)}{|\vec{r}-\vec{r}|} \mathrm{d}^{3} r \prime+a|\vec{r}|,
$$

where $\rho(r)$ is the density profile at radius $r$. The corresponding rotation velocity yields

$$
v^{2}(r)=v_{\mathrm{N}}^{2}(r)+v_{R}^{2}(r)
$$

where the subindex "N" stands for the Newtonian contribution and the Rindler circular velocity is given by

$$
v_{R}^{2}(r) \equiv a|\vec{r}|
$$

A similar approach to Eq. (3) was presented in Ref. [22] to fit rotation curves within a conformal gravity theory; see Refs. [23, 24] for further developments of this model. As emphasized in Ref. [5], the Rindler term constitutes a rough model which casts doubts on the description of rotation curves with such a linear growing of the velocity with the radius. It was therefore suggested to consider some kind of $r$-dependent term in the acceleration to test a more general Rindler hypothesis. Accordingly, we will assume below a power-law dependence that is a next step in complexity that adds an extra parameter, the exponent $n$ in Eq. (8) below. One may wonder how many extra parameters one might need to fit the data, and whether this represents a better fit than standard dark matter models. For instance, typical dark matter halos, e.g. NFW's [25, 26] or Burkert's [27] profiles, have two free parameters, a scale radius and density parameter. Then, from a phenomenological point of view, having two parameters in a dark matter alternative seems not too demanding, since it has the same number of free parameters to perform the fits. At the end, the best fits of the alternative model should be compared with the ones of the standard dark matter models, as it will be done at the end of the results section below. Let us remark that in standard dark matter profiles one expects some variation of the two free parameters from galaxy to galaxy, but this does not happen in Grumiller's models since the two free parameters are constants in every galaxy; $a$ is fundamental constant of nature and exponent $n$ is also a constant, yet to be determined. Therefore, the challenge to fit is bigger in Grumiller's models, and this is a price paid to modify gravity laws.

The main luminous components in a typical spiral galaxy are gas, stars, and a bulge, which are orbiting around the galactic center, and normally a dark matter halo is assumed to account for the high speed of the rotation curves. In the model used in the present work there exists no dark matter, but instead we use a Rindler term to explain the velocity distribution of stars and gas. 
Accordingly, the model developed here considers spherical symmetry and physical quantities will be independent of the polar angle. Therefore, the Newtonian contribution from stars $(\star)$ and gas $(G)$ can be thought as given by a spherized disk. The contribution from stars is then given by the Freeman disk [28, 29]:

$$
\rho_{\star}(r)=\frac{M_{d}}{2 \pi r_{d}^{2}} e^{-r / r_{d}},
$$

where $M_{d}$ is the mass of the disk and $r_{d}$ its radius. Thus, the rotation curve contribution from stars with a standard Newtonian dynamics yields [30]

$$
v_{\star}^{2}(r)=\frac{G M_{d}}{2 r_{d}}\left(\frac{r}{r_{d}}\right)^{2}\left[\mathrm{I}_{0}\left(\frac{r}{2 r_{d}}\right) \mathrm{K}_{0}\left(\frac{r}{2 r_{d}}\right)-\mathrm{I}_{1}\left(\frac{r}{2 r_{d}}\right) \mathrm{K}_{1}\left(\frac{r}{2 r_{d}}\right)\right],
$$

where the functions I and $\mathrm{K}$ are the modified Bessel functions; more details on the treatment of stars are given in Sec. IVA. On the other hand, the gas contribution $\left(v_{G}^{2}\right)$ is computed by integrating its surface brightness as in standard Newtonian lore, as we will explain in Sec. IVB.

Bringing together all contributions to the total $(T)$ rotation curve and including a generalized Rindler (GR) term,

$$
v_{T}^{2}(r)=\Upsilon_{\star} v_{\star}^{2}+v_{G}^{2}+v_{G R}^{2}(r),
$$

where $\Upsilon_{\star}$ is the mass-to-light ratio and

$$
v_{G R}^{2}(r) \equiv a|\vec{r}|^{n} .
$$

The case $n=1$ is the original model of modified gravity at large distances [5], as in Eqs. (2, 3). The new free parameters of the model of galactic rotation curves are $a$ and $n$, and they have to be determined by observations, instead of the two free parameters of the standard cold dark matter galactic profile, such as in NFW [25, 26], or alternative the Burkert [27], pseudoisothermal, or bound dark matter (BDM) [31] profiles; for a comparison of these profiles see Refs. [32, 33]. To extract information for the Rindler parameters, as an input we will need the total, observational rotation curve $v_{T}$, the rotation curve of the stellar component $v_{\star}$, and the gas component $v_{G}$. Following, we describe the observational data used and the models behind each component.

\section{THE THINGS GALACTIC SAMPLE}

We make use of the HI data provided by THINGS, which possess high resolution velocity fields of rotation curves that are ideal to test new dark matter profiles [32, 33] or, as it is our present case, to test new gravitational theories. THINGS galaxies have an observing data sample of 34 nearby galaxies containing a large range of luminosities and Hubble types, but we limit our sample to 17 low luminous (early type and dwarf) galaxies with smooth, symmetric and extended to large radii rotation curves and small or no bulge, see Table I. These properties can provide a good estimate to alternative models because their underlying dynamics is believed to be dominated by dark matter - or new alternative approaches- over all other components at all radii. For technical details and systematic effects treatment of the observations of the THINGS sample refer to Refs. [14, 34, 35]. For a complete analysis of its rotation curves, see Refs. [15, 33]. 
In addition to the rotation curves extracted from THINGS data and we use the $3.6 \mu \mathrm{m}$ data from Spitzer Infrared Nearby Galaxies Survey (SINGS) [36]. We follow the analysis of Refs. [15, 37] for the sample considered here.

\begin{tabular}{ccccc}
\multicolumn{5}{c}{ THINGS DATA } \\
\hline \hline Galaxy & $\begin{array}{c}\text { Distance } \\
(1)\end{array}$ & $R_{d}$ & $\log _{10} M_{\text {star }}$ & $\log _{10} M_{\text {star }}$ \\
N 925 & 9.2 & 3.30 & 10.01 & $(5)$ \\
N 2366 & 3.4 & 1.76 & 8.41 & 9.86 \\
N 2403 & 3.2 & 1.81 & 9.67 & 8.26 \\
N 2841 & 14.1 & 4.22 & 11.04 & 9.52 \\
N 2903 & 8.9 & 2.40 & 10.15 & 10.88 \\
N 2976 & 3.6 & 0.91 & 9.25 & 10.0 \\
N 3031 & 3.6 & 1.93 & 10.84 & 9.10 \\
N 3198 & 13.8 & 3.06 & 10.45 & 10.69 \\
N 3521 & 10.7 & 3.09 & 11.09 & 10.30 \\
N 3621 & 6.6 & 2.61 & 10.29 & 10.94 \\
N 4736 & 4.7 & 1.99 & 10.27 & 10.14 \\
N 5055 & 10.1 & 3.68 & 11.09 & 10.12 \\
N 6946 & 5.9 & 2.97 & 10.77 & 10.94 \\
N 7331 & 14.7 & 2.41 & 11.22 & 10.62 \\
N 7793 & 3.9 & 1.25 & 9.44 & 11.07 \\
I 2574 & 4.0 & 2.56 & 9.02 & 9.29 \\
D 154 & 4.3 & 0.72 & 7.42 & 8.87 \\
& & & & 7.27
\end{tabular}

TABLE I: Sample of THINGS late-type and dwarf galaxies as presented in Walter et al [14]. Columns: (1) Galaxy's name. (2) Distance to the galaxy in Mpc. (3) Characteristic radius of the stellar disk in kpc as given in Ref. [15]. (4) Logarithm of the stellar mass disk when considering the diet-Salpeter IMF in solar masses $\left(M_{\odot}\right)$, and (5) Logarithm of the stellar mass disk when considering the Kroupa IMF $\left(M_{\odot}\right)$.

\section{TREATMENT OF GAS AND STARS}

Our galactic model includes the two main observable components of a spiral galaxy: thin gaseous disk and a thick stellar disk. In most cases the stellar disk can be well described by a single exponential disk, given by Eq. (5). When necessary, in a small number of galaxies we have considered an additional central component, a bulge, containing a small fraction of the total luminosity of the galaxy, as described by Ref. [15].

Following, we describe in more detail the treatment for the stellar component in Sec. IVA, as well for the gas component in Sec. IVB.

\section{A. Stellar distribution}

To model the stellar disk when the $\Upsilon_{\star}$ is assumed to be constant at all radii, we use the approximation of a radial exponential profile of zero thickness, the Freeman disk [28, 29], since the disk vertical scale height does not change appreciably with radius and the correction to the velocity is around 5\% in most cases [38]. Then, the central surface density is given by Eq. (5), where $r_{d}$ is the scale length of the disk and $\Sigma_{0} \equiv \frac{M_{d}}{2 \pi r_{d}^{2}}$ is the central surface density with units $\left[M_{\odot} \mathrm{pc}^{-2}\right]$. These two parameters are obtained first by fitting the observed surface brightness profile, extracted 
from the SINGS images at the $3.6 \mu \mathrm{m}$ band and synthesized by Ref. [15], to the linear formula $\mu(R)=\mu_{0}+1.0857 r / r_{d}$, where $\mu_{0}$ is the central surface brightness given in observational units (mag $\operatorname{arcsec}^{-2}$ ), and $\mu_{0}$ and the surface brightness are related by a simple change of units. We get the surface density thanks to the mass-to-light ratio $\Upsilon_{\star}$, the standard additional free parameter in the mass model, introduced because we generally can only measure the distribution of the light instead of the mass. Stellar disks sometimes show radial color gradients, and it is believed that this provides an indication of stellar population between the inner and outer regions of a galaxy and produce $\Upsilon_{\star}$ gradients between these two regions of the disk [39]. We take the $\Upsilon_{\star}$ as a function of the radius in order to consider the different star's contribution as it depends on the region that we are analyzing.

When we estimate the Rindler parameters $(a, n), \Upsilon_{\star}$ is an important source of uncertainty, because these parameters are degenerate through Eq. (77). However, because stars have a major contribution near the center of the galaxy and the Rindler acceleration should contribute at large distance, we expect that $\Upsilon_{\star}$ does not significantly contribute to the uncertainties of the Rindler parameters. In the results section, we will show this is the case for most of the galaxies.

The $\Upsilon_{\star}$ has been modeled, e.g. in Salpeter [40], Kroupa [41], and Bottema [42], but the precise value for an individual galaxy is not well known and depends on extinction, star formation history, initial mass function (IMF), among others. Some assumptions have to be made respect to $\Upsilon_{\star}$ in order to reduce the number of free parameters in the model. In our case we studied three different $\Upsilon_{\star}$ models: (i) we obtained $\Upsilon_{\star}$ from the galaxy colors as predicted by spectrophotometric models with Kroupa [41] initial mass function (IMF) which is based on stellar population studies in the Milky Way and it yields low disk masses that minimizes the baryonic contribution; (ii) we determine $\Upsilon_{\star}$ through a diet-Salpeter [40], in which stellar mass population syntheses have proved [43] to maximize the disk mass contribution; and (iii) we assume the standard method in which the stellar $\Upsilon_{\star}$ is a model-independent free parameter. The resulting mass models reproduce well the rotation curves, and the Rindler parameters are derived within a reasonable uncertainty.

In our fittings of Kroupa and diet-Salpeter models we considered radial color gradients, but for the free mass model we consider a $\Upsilon_{\star}$ as an unknown, constant parameter to be determined. In all three models we took into account the bulge, when present, to contribute to the stellar disk.

The contribution of the atomic gas is considered in all three stellar Kroupa, diet-Salpeter, and free mass models, and it is briefly described as follows.

\section{B. Neutral gas distribution}

For the gas we assumed an infinitely thin disk in order to compute the corresponding rotation curve. For more technical details we refer to Ref. [15]. We point out that the case of a disk with sufficient central depression in the mass distribution can yield a net force pointing outwards, and this generates an imaginary rotation velocity and therefore a negative $v_{G}^{2}$. An imaginary velocity is just a reflect of the effective force of a test particle caused by a nonspherical mass distribution with a depression mass in the center. We have not included the contribution of the molecular gas since its surface density is only a few percent of that of the stars, therefore its contribution is reflected in a small increase in $\Upsilon_{\star}$ [44]. 


\section{RESULTS}

In order to constrain the Rindler modification of gravity we considered two scenarios. First, we treated the original Rindler model with $n=1$ and, second, the power-law dependence Rindler model, as suggested in Ref. [4], with two free parameters $(a, n)$, as explained in Sec. II]. For both cases we considered the Kroupa minimal disk and the diet-Salpeter maximum disk. In the third place, we present the fittings for Rindler models with $n=1$ and $n \neq 1$ using the Free $\Upsilon_{\star}$ stellar mass function.

We make use of the observed rotation curve, stellar, and gas components as an input for the numerical code, in order to obtain the Rindler parameters. To fit the observational velocity curve with the theoretical model we employ the $\chi^{2}$ goodness-of-fit test ( $\chi^{2}$ test $)$, that tells us how "close" are the theoretical to the observed values. In general the $\chi^{2}$ test statistics are of the form

$$
\chi^{2}=\sum_{i=1}^{n}\left(\frac{v_{\mathrm{obs}_{i}}-v_{\operatorname{model}_{i}}(r, a, n)}{\sigma_{i}}\right)^{2},
$$

where $\sigma$ is the standard deviation, and $n$ is the number of observations. One defines the reduced $\chi_{\text {red }}^{2} \equiv \chi^{2} /(\mathrm{n}-p-1)$, in which $\mathrm{n}$ is the number of observations and $p$ is the number of fitted parameters.

\section{A. Fitting the standard $(n=1)$ Rindler model with Kroupa and diet-Salpeter IMF}

We considered the original Rindler model with $n=1$ and proceed to fit the parameter $a$ for the Kroupa minimal disk and diet-Salpeter maximum disk with a $\chi^{2}$ test. When we minimize $\chi^{2}$ we use different methods (differential evolution, Nelder-Mead, and simulated annealing) to guarantee not to be in a local minimum. At the same time, we assume priors on the free parameter in order to have values greater than or equal to zero to obtain physical reasonable values. The results are presented in Table II, where the $\chi_{\text {red }}^{2}$ values are given. Notice that the different stellar mass models do not significantly change the determined value of the Rindler acceleration for most of the galaxies. The uncertainties in the rotation velocity are reflected in the uncertainties in the model parameters but in general these are small. One observes some spread in the values for $a$ (in units of $\mathrm{cm} / \mathrm{s}^{2}$ ), ranging from $0.93_{-0.44}^{+0.01}$ for $\mathrm{N} 2366$ to $9.57_{-0.06}^{+0.06}$ for $\mathrm{N} 2841$ in Kroupa's model, to account for a difference of an order of magnitude, but the uncertainties are small and they do not account for such a difference. In addition to this discrepancy, the fits to some of the galaxies present very

high $\chi_{\text {red }}^{2}$ values, that speaks for a poor fitting. In the diet-Salpeter mass model, $a$ varies in a similar fashion, given $0.62_{-0.10}^{+0.10}$ for N 3031 and $7.79_{-0.06}^{+0.06}$ for N 284, and again the goodness-of-fit test is not satisfactory for most of the galaxies. By comparing both fits, Kroupa did better in 9 (out of 17 ) cases and diet-Salpeter in 8 . None of the fits with $n=1$ had a $\chi_{\text {red }}^{2} \leq 1$.

\section{B. Fitting the power-law generalized Rindler model with Kroupa and diet-Salpeter IMF}

We now considered a Rindler model with power-law dependence, as suggested in Ref. [4], with two free parameters $(a, n)$ and we used the same stellar and gas models. The results are shown 


\begin{tabular}{r|rrr|rrr}
\multicolumn{7}{c}{ Rindler $n=1$} \\
\hline Galaxy & \multicolumn{7}{|c|}{ Kroupa } & diet-Salpeter & \\
\hline D 154 & $358.45_{-5.49}^{+5.45}$ & $1.16_{-0.02}^{+0.02}$ & 2.10 & $355.64_{-5.48}^{+5.45}$ & $1.15_{-0.02}^{+0.02}$ & 2.03 \\
I 2574 & $297.35_{-6.97}^{+6.91}$ & $0.96_{-0.02}^{+0.02}$ & 4.74 & $272.61_{-6.97}^{+6.91}$ & $0.88_{-0.02}^{+0.02}$ & 5.53 \\
N 2366 & $285.45_{-134.51}^{+1.94}$ & $0.93_{-0.44}^{+0.01}$ & 3.48 & $285.45_{-99.97}^{+2.66}$ & $0.93_{-0.32}^{+0.01}$ & 3.29 \\
N 2403 & $1258.65_{-5.78}^{+5.77}$ & $4.08_{-0.02}^{+0.02}$ & 11.80 & $1225.23_{-5.76}^{+5.75}$ & $3.97_{-0.02}^{+0.02}$ & 9.29 \\
N 2841 & $2952.36_{-19.50}^{+19.45}$ & $9.57_{-0.06}^{+0.06}$ & 76.10 & $2405.18_{-18.96}^{+18.91}$ & $7.79_{-0.06}^{+0.06}$ & 44.30 \\
N 2903 & $1985.81_{-13.96}^{+13.91}$ & $6.44_{-0.05}^{+0.05}$ & 71.80 & $1998.74_{-13.97}^{+13.92}$ & $6.48_{-0.05}^{+0.05}$ & 74.10 \\
N 2976 & $1000.00_{-723.12}^{+2.64}$ & $3.24_{-2.34}^{+0.01}$ & 8.05 & $698.04_{-44.80}^{+44.46}$ & $2.26_{-0.15}^{+0.14}$ & 10.70 \\
N 3031 & $2617.86_{-32.77}^{+32.68}$ & $8.48_{-0.11}^{+0.11}$ & 9.54 & $191.44_{-29.62}^{+29.52}$ & $0.62_{-0.10}^{+0.10}$ & 22.80 \\
N 3198 & $632.49_{-5.87}^{+5.85}$ & $2.05_{-0.02}^{+0.02}$ & 14.00 & $567.72_{-5.80}^{+5.78}$ & $1.84_{-0.02}^{+0.02}$ & 11.50 \\
N 3521 & $684.59_{-36.59}^{+36.21}$ & $2.22_{-0.12}^{+0.12}$ & 6.14 & $562.41_{-35.50}^{+35.10}$ & $1.82_{-0.12}^{+0.11}$ & 7.54 \\
N 3621 & $889.79_{-7.00}^{+6.98}$ & $2.88_{-0.02}^{+0.02}$ & 11.20 & $777.46_{-6.88}^{+6.86}$ & $2.52_{-0.02}^{+0.02}$ & 3.29 \\
N 4736 & $1005.43_{-28.11}^{+27.96}$ & $3.26_{-0.09}^{+0.09}$ & 20.50 & $420.13_{-27.24}^{+27.07}$ & $1.36_{-0.09}^{+0.09}$ & 8.74 \\
N 5055 & $559.90_{-8.74}^{+8.71}$ & $1.81_{-0.03}^{+0.03}$ & 5.09 & $399.76_{-8.34}^{+8.30}$ & $1.30_{-0.03}^{+0.03}$ & 15.80 \\
N 6946 & $1650.37_{-16.55}^{+16.51}$ & $5.35_{-0.05}^{+0.05}$ & 1.29 & $908.03_{-16.17}^{+16.12}$ & $2.94_{-0.05}^{+0.05}$ & 4.42 \\
N 7331 & $1938.02_{-24.55}^{+24.45}$ & $6.28_{-0.08}^{+0.08}$ & 8.65 & $1505.92_{-24.00}^{+23.90}$ & $4.88_{-0.08}^{+0.08}$ & 29.60 \\
N 7793 & $1720.65_{-358.31}^{+1.57}$ & $5.58_{-1.16}^{+0.01}$ & 10.50 & $1688.18_{-15.34}^{+37.17}$ & $5.47_{-0.05}^{+0.12}$ & 5.52 \\
N 925 & $612.29_{-14.39}^{+14.40}$ & $1.98_{-0.05}^{+0.05}$ & 3.74 & $453.50_{-14.36}^{+14.26}$ & $1.47_{-0.05}^{+0.05}$ & 5.72
\end{tabular}

TABLE II: Fits for the fixed power $n=1$ using the Kroupa and diet-Salpeter mass models. In columns (2) and (5) the Rindler acceleration has units of $\frac{\mathrm{km}^{2}}{\mathrm{~s}^{2} \mathrm{kpc}}$ and in columns (3) and (6) in units $\frac{\mathrm{cm}}{\mathrm{s}^{2}} \times 10^{-9}$.

in Table III. Now, the values of $a$ are given in the units $\left(\frac{m^{2}}{\mathrm{~s}^{2} k p c^{n}}\right)$ in consistency with the given $n$ values; one could extract an acceleration parameter here if one defines $a r^{n} \equiv a_{\text {new }} r\left(r / r_{\text {new }}\right)^{n-1}$, but we would only add an extra parameter $\left(r_{\text {new }}\right)$ that is completely degenerated with $a_{\text {new }}$.

The results of the fittings show a broader spread in the $a$ values. Again, the different stellar mass models do not significantly change the determined value of the Rindler acceleration or power-law exponent for most of the galaxies. By considering the Kroupa model one has $a=77.96_{-1.75}^{+1.74}$ for IC2574, and $a=32294.30_{-212.01}^{+211.39}$ for N 2903, accounting for 2 orders of magnitude in difference. For this IMF model, the variation on $n$ ranges from values less than 0.002 for $\mathrm{N} 4736$ to $2.14_{-0.044}^{+0.045}$ for N 2976, that yields a 3 orders of magnitude difference. Furthermore, the overall fitting results are again not quite satisfactory since some $\chi_{\text {red }}^{2}$ values are high. The analysis of the diet-Salpeter mass model shows a similar behavior: the "acceleration" parameter ranges from $2.12_{-0.12}^{+0.12}$ for $\mathrm{N}$ 3031 to $4216.82_{-176.98}^{+176.24}$ for $\mathrm{N} 4736$, whereas the power-law exponent goes from very small values $(<0.003)$ for N 4736 to $3.43_{-0.001}^{+2.196}$ for N 2976. For these IMF models the deviations of the Rindler parameters are a few orders of magnitude different. In Figs. 1 and 2 we present the contour plots of the Rindler parameters $(a, n)$ for $1 \sigma$ and $2 \sigma$ for both stellar models.

By comparing both Kroupa and diet-Salpeter fits, the former did much better in 14 (out of 17) cases and the later in only 3. However, the goodness-of-fit test does not render acceptable results since some galaxies present very high $\chi_{\text {red }}^{2}$ values, and only three of them have fits with $\chi_{\text {red }}^{2}<1$ 
(all for the Kroupa IMF model). Moreover, by considering only these three best fits, the most favored values of $a$ and $n$ are excluded from each other within various standard deviations.

Now, by comparing the best fits of Tables II and III it is clear that the generalized Rindler model fits better for all the galaxies.

\begin{tabular}{|c|c|c|c|c|c|c|}
\hline \multirow[b]{2}{*}{ Galaxy } & \multicolumn{3}{|c|}{ Kroupa } & \multicolumn{3}{|c|}{ diet-Salpeter } \\
\hline & $a$ & $n$ & $\chi_{\text {red }}^{2}$ & $a$ & $n$ & $\chi_{\text {red }}^{2}$ \\
\hline D 154 & $429.25_{-6.56}^{+6.52}$ & $0.87_{-0.010}^{+0.010}$ & 1.77 & $420.27_{-6.47}^{+6.43}$ & $0.88_{-0.010}^{+0.010}$ & 1.75 \\
\hline I 2574 & $77.96_{-1.75}^{+1.74}$ & $1.72_{-0.011}^{+0.011}$ & 0.98 & $53.57_{-1.29}^{+1.28}$ & $1.87_{-0.011}^{+0.011}$ & 1.32 \\
\hline N 2366 & $340.76_{-16.42}^{+16.15}$ & $1.02_{-0.028}^{+0.029}$ & 3.27 & $285.45_{-11.09}^{+18.13}$ & $1.13_{-0.009}^{+0.100}$ & 3.23 \\
\hline N 2403 & $4667.30_{-21.01}^{+20.97}$ & $0.48_{-0.002}^{+0.002}$ & 2.27 & $4000.25_{-18.50}^{+18.46}$ & $0.53_{-0.002}^{+0.002}$ & 2.55 \\
\hline N 2841 & $59071.70_{-334.49}^{+333.82}$ & $0.04_{-0.002}^{+0.002}$ & 0.90 & $38957.50_{-263.27}^{+262.73}$ & $0.13_{-0.002}^{+0.002}$ & 1.23 \\
\hline N 2903 & $32294.30_{-212.01}^{+211.39}$ & $0.03_{-0.002}^{+0.002}$ & 7.99 & $33026.10_{-215.67}^{+215.04}$ & $0.02_{-0.002}^{+0.002}$ & 8.34 \\
\hline N 2976 & $796.89_{-26.15}^{+25.89}$ & $2.14_{-0.044}^{+0.045}$ & 1.15 & $386.08_{-0.34}^{+336.71}$ & $3.43_{-0.001}^{+2.196}$ & 10.60 \\
\hline N 3031 & $9840.04_{-117.17}^{+116.91}$ & $0.36_{-0.006}^{+0.006}$ & 5.12 & $2.12_{-0.12}^{+0.12}$ & $3.34_{-0.023}^{+0.024}$ & 20.30 \\
\hline N 3198 & $2830.13_{-25.54}^{+25.46}$ & $0.54_{-0.003}^{+0.003}$ & 7.77 & $1531.59_{-15.42}^{+15.37}$ & $0.69_{-0.003}^{+0.003}$ & 9.54 \\
\hline N 3521 & $195.76_{-10.44}^{+10.31}$ & $1.42_{-0.016}^{+0.017}$ & 5.82 & $48.00_{-2.89}^{+2.85}$ & $1.83_{-0.018}^{+0.019}$ & 6.84 \\
\hline N 3621 & $3728.60_{-28.17}^{+28.09}$ & $0.50_{-0.003}^{+0.003}$ & 0.87 & $1880.38_{-16.34}^{+16.30}$ & $0.70_{-0.003}^{+0.003}$ & 1.24 \\
\hline N 4736 & $8377.42_{-177.79}^{+176.93}$ & $<0.002$ & 5.76 & $4216.82_{-176.98}^{+176.24}$ & $<0.003$ & 4.11 \\
\hline N 5055 & $322.28_{-5.06}^{+5.04}$ & $1.16_{-0.004}^{+0.004}$ & 4.96 & $11.81_{-0.24}^{+0.24}$ & $2.01_{-0.005}^{+0.000}$ & 13.50 \\
\hline N 6946 & $2005.70_{-20.07}^{+20.02}$ & $0.92_{-0.004}^{+0.004}$ & 1.25 & $57.86_{-1.00}^{+1.00}$ & $2.11_{-0.007}^{+0.007}$ & 2.47 \\
\hline N 7331 & $876.99_{-11.15}^{+11.11}$ & $1.27_{-0.004}^{+0.004}$ & 8.21 & $80.47_{-1.27}^{+1.26}$ & $1.99_{-0.005}^{+0.005}$ & 26.00 \\
\hline N 7793 & $2850.27_{-35.91}^{+35.74}$ & $0.69_{-0.009}^{+0.009}$ & 4.48 & $1884.05_{-26.74}^{+26.61}$ & $0.92_{-0.009}^{+0.009}$ & 5.31 \\
\hline N 925 & $100.24_{-2.32}^{+2.31}$ & $1.83_{-0.010}^{+0.010}$ & 1.35 & $20.43_{-0.60}^{+0.60}$ & $2.41_{-0.012}^{+0.012}$ & 2.30 \\
\hline
\end{tabular}

TABLE III: Fitted values for the Kroupa and diet-Salpeter stellar mass models with a power-law $\left(r^{n}\right)$ dependence on the Rindler acceleration. In column (2) and (5) we present the result for the Rindler acceleration (in units $\left.\frac{m^{2}}{\mathrm{~s}^{2} k p c^{n}}\right)$, in column (3) and (6) we present the power-law value of $r^{n}$. 


\section{Fitting the standard and power-law generalized Rindler model with the Free $\Upsilon_{\star}$ stellar model}

Our results above show that for some galaxies the estimated parameters and/or their associated $\chi_{\text {red }}^{2}$ present large discrepancies when we change the stellar mass model (Kroupa and diet-Salpeter), see e.g. galaxies N 3031 and N 6946 in Table III. Indeed, such results depend on the understanding on the stellar/baryonic physics in galaxies, and there is an extensive research in the field [40 43]. Because we do not completely comprehend the baryonic physics behind the galaxies we include the stellar "free mass model" in which the parameter $\Upsilon_{\star}$ is let free and get determined as a best fit, together with the Rindler parameters. In the few cases when a bulge was present we treated the bulge mass $\left(M_{B}\right)$ as a free parameter too, as described in Ref. [15]. Again, we started with the standard Rindler exponent $(n=1)$ and later considered $n$ free. The results are shown in Table IV. The standard Rindler model yields values for $a$ (in $\mathrm{cm} / \mathrm{s}^{2}$ ) varying from less than 0.1 for $\mathrm{N}$

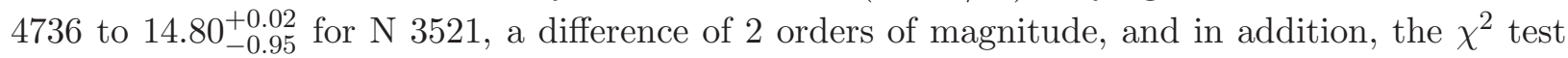
does not render satisfactory results for some of the galaxies. Then, we considered the generalized Rindler model to obtain the "acceleration" parameter ranging from < 10 for N 2366 to $19022_{-154}^{+153}$ for $\mathrm{N} 4736$, accounting for a 3 orders of magnitude difference. On the other hand, the power-

law exponent varies from $<0.002$ for N 3031 to $1.52_{-0.009}^{+0.009}$ for I 2574, a difference of 2 orders of magnitude. In Fig. 3 we present contour plots of the Rindler parameters $(a, n)$ for $1 \sigma$ and $2 \sigma$ for the free mass model.

By comparing both fits $(n=1$ vs $n \neq 1)$, one observes that both the $\chi_{\text {red }}^{2}$ and Rindler acceleration values substantially change. The generalized model achieved a better fit than the standard Rindler model for all the galaxies. Now, comparing the best fits of Tables III and IV i.e., generalized Rindler model with Kroupa vs free mass, the results are better in 14 cases (out of 17) for the free mass model. The price paid is that the free stellar model in the generalized Rindler model has a variation of the exponent $(n)$ of 3 orders of magnitude against a variation of only 2 orders of magnitude in the Kroupa model.

In a very recent work a similar model is considered [21] to fit the same eight (out of our seventeen) galaxies fixing $n=1$ and letting $\Upsilon_{\star}$ to be free. The authors fitted the following galaxies: N 2403, N 2841, N 2903, N 3198, N 3521, N 5055, N 7331, and D 154. However, they have not taken into account color gradients nor bulges, as we had, so their conclusions are not expected to be identical to ours. They concluded that six of these galaxies tend fit well to the data and that there is a preferred Rindler acceleration parameter of around $a \approx 3.0 \times 10^{-9} \mathrm{~cm} / \mathrm{s}^{2}$; they later fixed this acceleration parameter and found acceptable fits for five galaxies, and furthermore, an additional free parameter let them fit two more galaxies. When we analyze these galaxies in our results in Table IV we reach similar conclusions on the fits (except for N 3521) and to a convergence to a similar Rindler acceleration within $1 \sigma$ confidence level. However, when one adds more galaxies to the analysis the spread in the acceleration blows up, as shown in Table IV. 
Free mass model

\begin{tabular}{|c|c|c|c|c|c|c|c|c|c|c|}
\hline \multirow[b]{2}{*}{ Galaxy } & \multicolumn{5}{|c|}{$n=1$} & \multicolumn{5}{|c|}{$n \neq 1$} \\
\hline & $a$ & $a\left[\frac{\mathrm{cm}}{\mathrm{s}^{2}}\right]$ & $M_{D}$ & $M_{B}$ & & $\omega$ & $n$ & $M_{D}$ & $M_{B}$ & $\chi_{\mathrm{rec}}^{2}$ \\
\hline D 154 & $2.91_{-5.50}^{+5.42}$ & $1.08_{-0.02}^{+0.02}$ & $7.96_{-7.07}^{+7.06}$ & & 1.78 & $378.75_{-6.05}^{+6.01}$ & $3_{-0.010}^{+0.010}$ & $\begin{array}{l}0 \\
0\end{array} 7.80_{-7.06}^{+7.06}$ & & 1.75 \\
\hline I 2574 & $65.24_{-6.95}^{+6.89}$ & $1.18_{-0.02}^{+0.02}$ & $<1$ & & 3.94 & $6.85_{-2.65}^{+2.63}$ & $2_{-0.009}^{+0.009}$ & $\begin{array}{ll}9 & 8.26_{-7.77}^{+7.76}\end{array}$ & & 1.0 \\
\hline N 2366 & $6.84_{-16.69}^{+16.41}$ & $0.57_{-0.05}^{+0.05}$ & $9.00_{-8.69}^{+7.12}$ & & 2.30 & $<10$ & $1.11_{-1.00}^{+1.00}$ & $\begin{array}{ll}0 & 9.31_{-7.92}^{+9.26}\end{array}$ & & 1.58 \\
\hline N 2403 & $7.22_{-0.32}^{+97.65}$ & $2.58_{-0.30}^{+0.32}$ & $10.2_{-6.36}^{+9.83}$ & $6.77_{-5.72}^{+5.80}$ & 4.88 & $3070.1_{-16.1}^{+16.1}$ & $0.59_{-0.002}^{+0.002}$ & ${ }_{2}^{2} 9.85_{-8.07}^{+8.07}$ & & 0.7 \\
\hline N 2841 & $82.6_{-16.73}^{+16.57}$ & $3.83_{-0.05}^{+0.05}$ & $11.4_{-9.08}^{+9.08}$ & & 1.08 & $61227_{-359}^{+358}$ & $0.02_{-0.002}^{+0.002}$ & $\begin{array}{ll}2 & 11.0_{-9.08}^{+9.08}\end{array}$ & & 0.1 \\
\hline N 2903 & $5.84_{-12.87}^{+12.81}$ & $3.13_{-0.04}^{+0.04}$ & $10.8_{-8.75}^{+8.74}$ & $7.43_{-6.47}^{+6.39}$ & 2.47 & $4686.8_{-52.3}^{+52.1}$ & $0.53_{-0.004}^{+0.004}$ & $\begin{array}{ll}4 & 10.7_{-8.75}^{+8.74}\end{array}$ & & 1.8 \\
\hline N 2976 & $46.5_{-21.12}^{+99.93}$ & $8.58_{-0.07}^{+0.32}$ & $5.86_{-4.95}^{+6.95}$ & & 2.32 & $2130.7_{-41.6}^{+41.2}$ & $1.30_{-0.030}^{+0.030}$ & $\begin{array}{ll}{ }_{0}^{0} & 8.38_{-7.59}^{+7.59}\end{array}$ & & 0.99 \\
\hline 031 & $02.5_{-3}^{+3}$ & $5.19_{-0.10}^{+0.10}$ & $10.8_{-8.50}^{+8.50}$ & $7.83_{-6.78}^{+6.79}$ & 93 & $34102_{-228}^{+233}$ & $<0.002$ & $210.5_{-8.51}^{+8.50}$ & & 8 \\
\hline 8 & $503.00_{-5.74}^{+5.72}$ & $1.63_{-0.02}^{+0.02}$ & $10.5_{-8.62}^{+8.62}$ & $7.96_{-6.97}^{+6.95}$ & 5.11 & $2144.6_{-21.0}^{+21.0}$ & $0.60_{-0.003}^{+0.003}$ & $\begin{array}{ll}3 \\
3\end{array} 10.4_{-8.62}^{+8.62}$ & $8.65_{-7}^{+}$ & 96 \\
\hline N 3521 & $4580.0_{-294}^{+7.67}$ & $14.80_{-0.95}^{+0.02}$ & $9.00_{-6.45}^{+6.88}$ & & 63.10 & $120.08_{-8.96}^{+6.75}$ & & $\begin{array}{ll}7 & 11.0_{-9.05}^{+8.96}\end{array}$ & & 1.78 \\
\hline N 3621 & $689.06_{-9.49}^{+4.84}$ & $2.23_{-0.03}^{+0.02}$ & $10.4_{-8.32}^{+8.39}$ & & 0.67 & $2015.0_{-17.4}^{+17.3}$ & $0.67_{-0.003}^{+0.003}$ & $\begin{array}{ll}{ }_{3}^{3} & 10.3_{-8.35}^{+8.35}\end{array}$ & & 0.56 \\
\hline N 4736 & $<10$ & $<0.1$ & $10.5_{-8.44}^{+8.44}$ & $7.27_{-6.24}^{+6.34}$ & 53.0 & $19022_{-154}^{+153}$ & $0.10_{-0.001}^{+0.001}$ & $\begin{array}{ll}{ }_{1}^{1} & 7.08_{-5.90}^{+7.90}\end{array}$ & & 32 \\
\hline N 5055 & $9_{-8.82}^{+8.75}$ & $0^{+0 .}$ & 1 & $<1$ & 9.30 & $27370_{-175}^{+175}$ & 02 & $\begin{array}{ll}2 & 10.5_{-8.76}^{+8.76}\end{array}$ & & 0.8 \\
\hline N 6946 & $78.7_{-16.30}^{+16.64}$ & $4.47_{-0.05}^{+0.05}$ & $10.7_{-8.54}^{+8.55}$ & $<1$ & 1.48 & $7052.8_{-53.6}^{+53.5}$ & $0.50_{-0.003}^{+0.003}$ & $\begin{array}{l}3 \\
3\end{array} 10.6_{-8.54}^{+8.54}$ & & 1.2 \\
\hline N 7331 & $07.9_{-24.73}^{+24.65}$ & $5.53_{-0.08}^{+0.08}$ & $11.0_{-9.01}^{+9.01}$ & $12.2_{-11.1}^{+11.2}$ & 0.26 & $3348.2_{-45.8}^{+45.7}$ & $0.79_{-0.005}^{+0.005}$ & $\begin{array}{ll}5 & 10.9_{-9.01}^{+9.01}\end{array}$ & $7.70_{-6.77}^{+6.67}$ & 0.2 \\
\hline 7793 & $1523.1_{-23.79}^{+23.79}$ & $4.94_{-0.08}^{+0.08}$ & $9.47_{-7.85}^{+7.85}$ & & 4.88 & $2277.8_{-30.4}^{+30.2}$ & $83_{-0.009}^{+0.009}$ & $\begin{array}{ll}{ }_{9}^{9} & 9.31_{-7.85}^{+7.85}\end{array}$ & & 4.61 \\
\hline N 925 & $803.09_{-450}^{+0.42}$ & $2.60_{-1.46}^{+0.01}$ & $7.55_{-6.88}^{+6.88}$ & & 4.07 & $420.11_{-7.17}^{+7.12}$ & $.33_{-0.008}^{+0.008}$ & $\begin{array}{ll}8 & 9.47_{-8.34}^{+8.34}\end{array}$ & & 1.1 \\
\hline
\end{tabular}

TABLE IV: Fits for free mass model. In columns (2), (3) and (7) we present the Rindler acceleration in units of $\frac{k m^{2}}{\mathrm{~s}^{2} k p c}, \mathrm{~cm} / \mathrm{s}^{2} \times 10^{-9}$ and $\frac{m^{2}}{\mathrm{~s}^{2} k p c^{n}}$, respectively. The stellar disk $\left(M_{D}\right)$ and bulge $\left(M_{B}\right)$ masses are given in $\log _{10}$ solar mass units.

\section{Comparison of Rindler models with dark matter profiles}

In order to compare the above results with those of standard dark matter profiles we include the fittings of the NFW [25, 26] and Burkert [27] profiles for the Kroupa and diet-Salpeter stellar mass models. The results are shown in Tables $\mathrm{V}$ and $\mathrm{VI}$, which were extracted from our previous work [33], where we employed the same galaxies and the fits were computed with the same technique as in the present work. In general, as found by many authors cored profiles fit better than cuspy profiles to the type of galaxies study here, see e.g. Refs [15 20, 32, 33]. This is evident from the lower $\chi_{\text {red }}^{2}$ values for Burkert's profile in comparison to NFW's for most of the galaxies for both stellar mass models.

To compare among the different models we constructed TableVII with the $\chi_{\text {red }}^{2}$ values for NFW, Burkert, standard Rindler with $n=1$, and generalized Rindler $n \neq 1$, for both Kroupa and diet-Salpeter stellar mass models. The results are as follows:

- As expected, the Rindler model with two free parameters $(a, n)$ fits better than the model with a single parameter $(a, n=1)$ for both Kroupa and diet-Salpeter stellar mass models, for all galaxies. 
- The standard Rindler model $(n=1)$ does fit worst than NFW's and Burkert's profiles for the Kroupa mass model, and the same trend is observed for the diet-Salpeter stellar model. In this later case, the standard Rindler model fits better than NFW and Burkert only for one galaxy (N 3521), and it fits better than Burkert for one galaxy (N 3621), and it fits equally as well as NFW for two galaxies (N 6946 and N 7331).

- Considering the generalized Rindler model $(n \neq 1)$ for the Kroupa stellar mass model the fits are better than the standard Rindler model, since one now has 2 degrees of freedom. This model fits better than both NFW and Burkert models for four galaxies (N 2841, N 2976, N 3521, and N 3621) and, in addition, it does fit better than NFW for four galaxies (IC2574, N 5055, N 7331, and N 925) and better than Burkert for one galaxy (N 7793) and it fits equally well as one galaxy ( $\mathrm{N}$ 3031). In all other cases, however, 20 of the 34 possibilities, NFW and Burkert profiles for the Kroupa stellar mass model fit better than the generalized Rindler model: The NFW profile fits better for 9 galaxies (out of 17) than the generalized Rindler model and Burkert's profile achieves a better fit for 11 galaxies (out of 17).

- The generalized Rindler model $(n \neq 1)$ for the diet-Salpeter stellar mass model results are slightly better than the Kroupa's model: The model fits better than both NFW and Burkert models for five galaxies (N 3031, N 3521, N 3621, N 6946, and N 925) and, in addition, it fits better than NFW for three galaxies (IC2574, N 5055, and N 7331), but it does not fit better for any other galaxy with Burkert's profile. Taking into account all the other cases, however, 21 of the 34 possibilities, NFW and Burkert profiles for the diet-Salpeter stellar mass model fit better than the generalized Rindler model: The NFW profile fits better for 9 galaxies (out of 17) and Burkert profile achieves a better fit for 12 galaxies (out of 17) than the generalized Rindler model.

For completeness, in Fig. (4) we show rotation curves of three galaxies (N 2841, N 3621, and N 6946) as examples of typical fits for the standard $(n=1)$ Rindler model and its comparison with NFW and Burkert profiles. For some of the galaxies (e.g. N 2841), the rotation curve linear term, given by Eq. 4, overestimates the external rotational curve given rise to bad fits, a point that was

warned in Ref. [4] and also discussed in Ref. [21]. On the contrary, it is argued, e.g. in Ref. [20], that rotation curves tend to slowly decrease after a few optical radius.

As we showed in Refs. [32, 33], the BDM profile fits even better than or at least equally as well as Burkert's and NFW's for the same galaxies considered here, but in order to avoid multiple comparisons that may be cumbersome, we have not included the BDM profile results here that are extensively discussed elsewhere [33]. By considering alternatives to dark matter profiles one would desire to accomplish a better phenomenology, as the BDM model does, but the Rindler modified gravity does not.

\section{CONCLUSIONS AND FINAL REMARKS}

We have tested the idea that a modification of the Newtonian potential stemming from a Rindler acceleration that modifies gravity, as proposed by Ref. [4], would account for the dark matter content in spiral galaxies. The theory yields an effective new term in the theoretical rotation curve 


\begin{tabular}{r|rrr|rrr}
\multicolumn{6}{c}{ Kroupa } \\
\hline & \multicolumn{2}{|c}{ NFW } & \multicolumn{3}{|c}{ Burkert } \\
Galaxy & $r_{s}$ & $\log \rho_{0}$ & $\chi_{\text {red }}^{2}$ & \multicolumn{2}{c}{$r_{s}$} & $\log \rho_{0} \chi_{\text {red }}^{2}$ \\
\hline D 154 & $15.14_{-0.18}^{+0.17}$ & $6.1_{-4.28}^{+4.28}$ & 1.06 & $2.47_{-0.02}^{+0.02}$ & $7.43_{-5.6}^{+5.61}$ & 0.43 \\
I 2574 & $>10^{6}$ & $<0.1$ & 2.4 & $14.74_{-0.54}^{+0.57}$ & $6.62_{-4.95}^{+4.96}$ & 0.69 \\
N 2366 & $15.47_{-0.57}^{+0.58}$ & $6.07_{-4.74}^{+4.75}$ & 3 & $1.75_{-0.05}^{+0.04}$ & $7.72_{-6.37}^{+6.37}$ & 1.34 \\
N 2403 & $10.38_{-0.03}^{+0.03}$ & $7.14_{-4.8}^{+4.8}$ & 0.82 & $3.86_{-0.01}^{+0.01}$ & $7.98_{-5.63}^{+5.63}$ & 1.6 \\
N 2841 & $6.67_{-0.02}^{+0.02}$ & $8.18_{-5.89}^{+5.89}$ & 1.29 & $4.08_{-0.01}^{+0.01}$ & $8.62_{-6.33}^{+6.34}$ & 3.02 \\
N 2903 & $4.92_{-0.02}^{+0.02}$ & $8.12_{-5.94}^{+5.94}$ & 3.43 & $3.06_{-0.01}^{+0.01}$ & $8.55_{-6.45}^{+6.37}$ & 2.01 \\
N 2976 & $>10^{5}$ & $1.77_{-0.15}^{+0.46}$ & 6.3 & $>10^{4}$ & $7.69_{-6.2}^{+6.19}$ & 1 \\
N 3031 & $8.35_{-0.06}^{+0.06}$ & $7.48_{-5.55}^{+5.56}$ & 4.96 & $3.5_{-0.03}^{+0.02}$ & $8.22_{-6.3}^{+6.29}$ & 5.12 \\
N 3198 & $20.95_{-0.11}^{+0.11}$ & $6.54_{-4.47}^{+4.5}$ & 4.75 & $8.1_{-0.04}^{+0.04}$ & $7.34_{-5.28}^{+5.29}$ & 2.49 \\
N 3521 & $>10^{6}$ & $1.03_{--0.28}^{+-0.27}$ & 5.66 & $15.70_{-0.56}^{+0.58}$ & $6.93_{-5.61}^{+5.61}$ & 5.21 \\
N 3621 & $17.1_{-0.08}^{+0.08}$ & $6.72_{-4.6}^{+4.6}$ & 1.45 & $5.77_{-0.02}^{+0.02}$ & $7.63_{-5.51}^{+5.51}$ & 5.59 \\
N 4736 & $0.23_{-0.02}^{+0.02}$ & $10.44_{-8.74}^{+8.74}$ & 1.34 & $0.22_{-0.05}^{+0.05}$ & $10.45_{-8.76}^{+8.76}$ & 1.3 \\
N 5055 & $>10^{6}$ & $<2$ & 5.09 & $23.54_{-0.26}^{+0.27}$ & $6.6_{-4.8}^{+4.79}$ & 4.11 \\
N 6946 & $96.74_{-0.85}^{+0.84}$ & $5.87_{-3.87}^{+3.87}$ & 1.21 & $8.12_{-0.06}^{+0.06}$ & $7.52_{-5.52}^{+5.52}$ & 1.14 \\
N 7331 & $>10^{6}$ & $<2$ & 8.63 & $17.4_{-0.19}^{+0.19}$ & $7.23_{-5.34}^{+5.33}$ & 7.06 \\
N 7793 & $8.62_{-0.08}^{+0.08}$ & $7.15_{-5.25}^{+5.26}$ & 4.07 & $1.88_{-0.02}^{+0.01}$ & $8.35_{-6.45}^{+6.44}$ & 7.88 \\
N 925 & $>10^{5}$ & $1.95_{-0.32}^{+0.34}$ & 3.68 & $21.15_{-0.95}^{+1.02}$ & $6.77_{-5.13}^{+5.14}$ & 1.19
\end{tabular}

TABLE V: Fitted parameter values for the NFW and Burkert profiles with a Kroupa mass stellar model.

of the form $v_{T}^{2}(r)=\Upsilon_{\star} v_{\star}^{2}+v_{G}^{2}+a|\vec{r}|^{n}$, where the last term would replace the contribution of the dark matter profile.

We have made use of the HI data provided by THINGS [14], which possess high resolution velocity fields of rotation curves that are ideal to test new dark matter profiles [32, 33] or, as in the present work, new gravity models. We have considered the gas component that is computed by integrating its surface brightness as in the standard Newtonian theory, three stellar mass models (Kroupa, dietSalpeter, and free mass), and the standard Rindler model $(n=1)$ and a generalized power-law $(n$ free). We have considered three stellar mass models since the galactic dynamics is encoded in the baryonic physics, and this is somewhat unknown. Therefore, the parameter determination of the modified gravity depends on the the understanding of the stellar/baryonic physics in galaxies, and to reach conclusions one has to be cautious, through finding best fits and looking for convergence of parameters' values.

The results of the fits are shown in Tables II, III, and IV for three stellar mass models for both Rindler models, $n=1$ and $n$ free. We showed, in Sec. $\mathrm{VA}$, that the fits for $n=1$ are poor, since most of the $\chi_{\text {red }}^{2}$ are bigger than unity. However, the most important problem is the Rindler acceleration parameter does not converge to a single value. The computed parameter is in the interval $0.93_{-0.44}^{+0.01}<a<9.57_{-0.06}^{+0.06}$ in Kroupa's model, to account for a difference of an order of magnitude, whereas $0.62_{-0.10}^{+0.10}<a<7.79_{-0.06}^{+0.06}$ in diet-Salpeter's model, in a similar fashion as in the previous model. In each stellar mass model the uncertainties in the Rindler acceleration are 


\begin{tabular}{|c|c|c|c|c|c|c|}
\hline \multirow[b]{2}{*}{ Galaxy } & \multicolumn{3}{|c|}{ NFW } & \multicolumn{3}{|c|}{ Burkert } \\
\hline & $r_{s}$ & $\log \rho_{0}$ & $\chi_{\text {red }}^{2}$ & $r_{s}$ & $\log \rho_{0}$ & $\chi_{\text {red }}^{2}$ \\
\hline D 154 & $15.95_{-0.19}^{+0.19}$ & $6.07_{-4.25}^{+4.25}$ & 1.09 & $2.53_{-0.03}^{+0.03}$ & $7.41_{-5.59}^{+5.59}$ & 0.39 \\
\hline I 2574 & $>10^{5}$ & $<2$ & 5.49 & $20.82_{-1.14}^{+1.27}$ & $6.51_{-4.89}^{+4.89}$ & 1.16 \\
\hline N 2366 & $15.99_{-0.62}^{+0.63}$ & $6.03_{-4.71}^{+4.74}$ & 2.94 & $1.78_{-0.05}^{+0.05}$ & $7.69_{-6.35}^{+6.36}$ & 1.34 \\
\hline N 2403 & $12.53_{-0.03}^{+0.03}$ & $6.99_{-4.65}^{+4.65}$ & 1.08 & $5.71_{-0.02}^{+0.02}$ & $7.63_{-5.32}^{+5.33}$ & 1.37 \\
\hline N 2841 & $5.84_{-0.43}^{+0.43}$ & $8.37_{-6.51}^{+6.51}$ & 0.49 & $3.85_{-0.03}^{+0.03}$ & $8.75_{-7.01}^{+7.02}$ & 0.93 \\
\hline N 2903 & $4.81_{-0.01}^{+0.01}$ & $8.15_{-5.96}^{+6.04}$ & 3.73 & $2.85_{-0.01}^{+0.01}$ & $8.63_{-6.43}^{+6.44}$ & 1.23 \\
\hline N 2976 & $>10^{5}$ & $2.41_{-1.22}^{+1.22}$ & 10.49 & $>10^{4}$ & $7.46_{-5.95}^{+6.41}$ & 5.11 \\
\hline N 3031 & $>10^{6}$ & $<2$ & 22.76 & $1.90_{-0.01}^{+0.01}$ & $9.06_{-7.81}^{+7.82}$ & 4.37 \\
\hline N 3198 & $41.1_{-0.27}^{+0.27}$ & $6.01_{-4.01}^{+4.01}$ & 7.86 & $9.74_{-0.05}^{+0.05}$ & $7.17_{-5.13}^{+5.16}$ & 0.93 \\
\hline N 3521 & $>10^{6}$ & $<2$ & 12.5 & $162_{-50.1}^{+135}$ & $6.22_{-5.01}^{+5.02}$ & 11.5 \\
\hline N 3621 & $43.81_{-0.27}^{+0.27}$ & $6.03_{-3.98}^{+3.98}$ & 1.44 & $9.41_{-0.05}^{+0.05}$ & $7.21_{-5.17}^{+5.15}$ & 3.64 \\
\hline N 4736 & $0.07_{-0.01}^{+0.01}$ & $11.54_{-10.16}^{+10.11}$ & 1.3 & $0.08_{-0.01}^{+0.01}$ & $11.34_{-9.96}^{+9.91}$ & 1.29 \\
\hline N 5055 & $>10^{6}$ & $<1$ & 15.74 & $2.52_{-0.03}^{+0.03}$ & $8.79_{-6.89}^{+6.89}$ & 3.47 \\
\hline N 6946 & $>10^{5}$ & $2.89_{-1.14}^{+1.15}$ & 4.42 & $309.22_{-104.69}^{+322.11}$ & $6.65_{-4.9}^{+4.89}$ & 2.49 \\
\hline N 7331 & $>10^{6}$ & $<2$ & 29.6 & $27.58_{-0.48}^{+0.49}$ & $7.00_{-5.14}^{+5.15}$ & 15.57 \\
\hline N 7793 & $30.08_{-0.36}^{+0.36}$ & $6.4_{-4.55}^{+4.55}$ & 5.01 & $3.09_{-0.03}^{+0.03}$ & $7.98_{-6.13}^{+6.14}$ & 4.3 \\
\hline N 925 & $>10^{6}$ & $1.23_{-0.27}^{+0.26}$ & 5.64 & $61.11_{-8.61}^{+18.46}$ & $6.54_{-5.23}^{+4.78}$ & 2.54 \\
\hline
\end{tabular}

TABLE VI: Fitted parameter values for the NFW and Burkert profiles with a diet-Salpeter mass stellar model.

small and they could not account for such a big spread in the intervals of $a$.

When the power-law parameter $n$ is set free, see Sec. VB and Table III, the fits become better, and by comparing them Kroupa's did much better in 14 (out of 17) cases than diet-Salpeter's. However, the goodness-of-fit test does not render acceptable results since some galaxies present very high $\chi_{\text {red }}^{2}$ values. Moreover, they again suffer from a broad spread in the determination of the Rindler parameter values, as shown in the contour plots of Fig. 11. For Kroupa's model the parameters are $77.96_{-1.75}^{+1.74}<a<32294.30_{-212.01}^{+211.39}$, accounting for 2 orders of magnitude in difference and $0.002 \sim n<2.14_{-0.044}^{+0.045}$ that yields a 3 orders of magnitude difference. For diet-Salpeter parameters the spreads are similar.

When the mass-to-light ratio is set free, see Sec. VC and Table IV, the fits are better than previous models when $n$ is a free parameter, but again the spread in parameters is high: $10 \sim a<$ $19022_{-154}^{+153}$, accounting for a 3 orders of magnitude difference and $0.002 \sim n<1.52_{-0.009}^{+0.009}$, a difference of 2 orders of magnitude, see also Fig. 3. A very recent work [21] considers the same problem and using the same eight (of our seventeen) galaxies for the standard Rindler model $(n=1)$, concluding that for six galaxies their results tend to converge to a single Rindler acceleration parameter. In our case, we observe this evidence too but when one takes into account more galaxies or other stellar galactic models their conclusions do not hold.

Finally, we have compared our previous results with two standard dark matter profiles, one cuspy (NFW) and one cored (Burkert), see Tables V. VI, and VII. The goodness-of-fit test favors first 


\section{Goodness-of-fit comparison table}

\begin{tabular}{|c|c|c|c|c|c|c|c|c|}
\hline \multirow{2}{*}{\begin{tabular}{l|} 
Galaxy \\
D 154
\end{tabular}} & NFW I & \multicolumn{3}{|c|}{$\begin{array}{l}\text { Kroupa } \\
\text { kert Rindler Rindler } \\
\quad(n=1)(n \neq 1)\end{array}$} & \multicolumn{4}{|c|}{$\begin{array}{l}\text { diet-Salpeter } \\
\text { NFW Burkert Rindler Rindler } \\
(n=1)(n \neq 1)\end{array}$} \\
\hline & 1.06 & 0.43 & 2.10 & 1.77 & 1.09 & 0.39 & 2.03 & 1.7 \\
\hline I 2574 & 2.40 & & & 0.98 & & & & 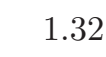 \\
\hline & 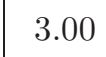 & & & 3.27 & & & & 3. \\
\hline & 0. & & & 27 & & & & \\
\hline & & & & 90 & & & & \\
\hline & & & & & & & & \\
\hline & & & & & & & & 20 \\
\hline & & & & 5.12 & & & & 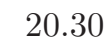 \\
\hline & & & & 77 & & & & . \\
\hline & & & & 82 & 12 & & & 0.0 \\
\hline & & & & 57 & & & & 1.2 \\
\hline & & & & 5.76 & & & & 4.1 \\
\hline & 5 & & & 4.96 & 15.74 & & & 10.0 \\
\hline & 1 & & & 1.25 & & & & 2.4 \\
\hline & 8.63 & & & 8.21 & 29.60 & 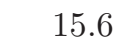 & 29.60 & 26.0 \\
\hline & 4.07 & & & 4.48 & & 4.30 & 5.52 & 5.3 \\
\hline N 925 & 3.68 & 1.19 & 3.74 & 1.35 & 5.64 & 2.54 & 5.72 & 2. \\
\hline
\end{tabular}

TABLE VII: Summary of the $\chi_{\text {red }}^{2}$ values for the different profiles with the Kroupa and diet-Salpeter stellar mass models.

Burkert and then NFW profiles over Rindler's modify gravity. The tendency is clearer for the standard Rindler $(n=1)$ that fits worse than both NFW's and Burkert's profiles for the Kroupa and diet-Salpeter stellar mass models. The generalized Rindler model $(n \neq 1)$ for the diet-Salpeter stellar mass model results are slightly better than the Kroupa's model, but still the NFW profile fits better for 9 galaxies (out of 17) and Burkert profile achieves a better fit for 12 galaxies (out of 17) than the generalized Rindler model.

The overall conclusion is that although the Rindler modified gravity fits are achievable for the considered galaxies, in many cases they show high $\chi_{\text {red }}^{2}$ values, and a high spread in the Rindler parameters $(a, n)$ that points for an inconsistent model. Furthermore, the standard dark matter profiles (NFW and Burkert) or the alternative BDM model do a better job to fittings of the rotation curves.

\section{ACKNOWLEDGMENTS}

We thank Professor Erwin de Blok for providing the observational data of THINGS. A.M. and J.M. acknowledge financial support from Conacyt Project No. 80519 and UNAM PAPIIT Project 
No. IN100612.

[1] J. Frieman, M. Turner and D. Huterer, Annu. Rev. Astron. Astrophys. 46, 385 (2008) arXiv:0803.0982 [astro-ph]].

[2] J. L. Cervantes-Cota and G. Smoot, AIP Conf. Proc. 1396, 28 (2011) arXiv:1107.1789 [astro-ph.CO]].

[3] T. Clifton, P. G. Ferreira, A. Padilla and C. Skordis, Phys. Rept. 513, 1 (2012) arXiv:1106.2476 [astro-ph.CO]].

[4] D. Grumiller, Phys. Rev. Lett. 105, 211303 (2010) [Erratum-ibid. 106, 039901 (2011)] arXiv:1011.3625 [astro-ph.CO]].

[5] D. Grumiller and F. Preis, Int. J. Mod. Phys. D 20, 2761 (2011) arXiv:1107.2373 [astro-ph.CO]].

[6] M. Milgrom, Astrophys. J. 270, 365 (1983).

[7] R. H. Sanders and S. S. McGaugh, Annu. Rev. Astron. Astrophys. 40, 263 (2002).

[8] B. Famaey and S. McGaugh, arXiv:1112.3960 [astro-ph.CO].

[9] J. Sultana and D. Kazanas, Phys. Rev. D 85, 081502 (2012).

[10] H. Culetu, Class. Quant. Grav. 29, 235021 (2012) arXiv:1202.4296 [gr-qc]].

[11] X. Li and Z. Chang, Commun. Theor. Phys. 57, 611 (2012) arXiv:1108.3443 [gr-qc]].

[12] L. Iorio, Mon. Not. Roy. Astron. Soc. 419, 2226 (2012) arXiv:1108.0409 [gr-qc]].

[13] S. Carloni, D. Grumiller and F. Preis, Phys. Rev. D 83, 124024 (2011) arXiv:1103.0274 [astro-ph.EP]].

[14] F.Walter et al., Astron. J. 136, 2563 (2008) arXiv:0810.2125].

[15] W. J. G. de Blok, F. Walter, E. Brinks, C. Trachternach, S. H. Oh and R. C. . Kennicutt, Astron. J. 136, 2648 (2008).

[16] K. Spekkens, R. Giovanelli, M. P. Haynes, Astron. J. 129, 2119 (2005).

[17] R. Kuzio de Naray, S. S. McGaugh, and W.J.G. de Blok, Astrophys. J. 676, 920 (2008).

[18] F. Donato, G. Gentile, P. Salucci, C. Frigerio Martins, M.I. Wilkinson, et al Mon. Not. Roy. Astron. Soc. 397, 1169 (2009); G. Gentile, P. Salucci, U. Klein, D. Vergani, P. Kalberla, Mon. Not. Roy. Astron. Soc. 351,903 (2004). P. Salucci, arXiv:1008.4344 [astro-ph.CO].

[19] G. Gentile, P. Salucci, U. Klein, D. Vergani and P. Kalberla, Mon. Not. Roy. Astron. Soc. 351, 903 (2004) arXiv:astro-ph/0403154.

[20] P. Salucci, A. Lapi, C. Tonini, G. Gentile, I. Yegorova and U. Klein, Mon. Not. Roy. Astron. Soc. 378, 41 (2007) arXiv:astro-ph/0703115.

[21] H. -N. Lin, M. -H. Li, X. Li and Z. Chang, arXiv:1209.3532 [astro-ph.CO].

[22] P. D. Mannheim, Astrophys. J. 479, 659 (1997) astro-ph/9605085.

[23] P. D. Mannheim, Prog. Part. Nucl. Phys. 56, 340 (2006) astro-ph/0505266].

[24] P. D. Mannheim and J. G. O'Brien, Phys. Rev. Lett. 106, 121101 (2011) arXiv:1007.0970 [astroph.CO]].

[25] J. F. Navarro, C. S. Frenk and S. D. M. White, Astrophys. J. 462, 563 (1996) arXiv:astro-ph/9508025.

[26] J. F. Navarro, C. S. Frenk and S. D. M. White, Astrophys. J. 490, 493 (1997) arXiv:astro-ph/9611107.

[27] A. Burkert, Astrophys. J. 447, L25 (1995) astro-ph/9504041.

[28] K. Freeman, Astrophys. J. 160, 811 (1970).

[29] J. N. Bahcall, R. M. Soneira, Astrophys. J. Suppl. Ser. 44, 73 (1980).

[30] J. Binney, S. Tremaine, Galactic Dynamics (Princeton, Univ. Press, NJ, 2008) 2nd ed.

[31] A. de la Macorra, Astropart. Phys. 33, 195 (2010) [arXiv:0908.0571 [astro-ph.CO]].

[32] A. de la Macorra, J. Mastache and J. L. Cervantes-Cota, Phys. Rev. D 84, 121301 (2011) arXiv:1107.2166 [astro-ph.CO]].

[33] J. Mastache, A. de la Macorra and J. L. Cervantes-Cota, Phys. Rev. D 85, 123009 (2012) arXiv:1107.5560 [astro-ph.CO]].

[34] C. Trachternach, W. J. G. de Blok, F. Walter, E. Brinks and R. C. . Kennicutt, Astron. J. 136, 2720 (2008) arXiv:0810.2116 [astro-ph]]. 
[35] S. H. Oh, W. J. G. de Blok, F. Walter, E. Brinks and R. C. . Kennicutt, Astron. J. 136, 2761 (2008) arXiv:0810.2119 [astro-ph]].

[36] R. C. . Kennicutt et al., Publ. Astron. Soc. Pac. 115, 928 (2003) arXiv:astro-ph/0305437.

[37] S. S. McGaugh, W. J. G. de Blok, J. M. Schombert, R. K. de Naray and J. H. Kim, Astrophys. J. 659, 149 (2007) arXiv:astro-ph/0612410.

[38] Burlak, A.N., Gubina, V.A., Tyurina, N.V., Astro. Lett. 23, 522 (1997).

[39] V. A. Taylor, R. A. Jansen, R. A. Windhorst, S. C. Odewahn and J. E. Hibbard, Astrophys. J. 630, 784 (2005) astro-ph/0506122.

[40] E. E. Salpeter, Astrophys. J. 121, 161 (1955).

[41] P. Kroupa, Mon. Not. Roy. Astron. Soc. 322, 231 (2001) arXiv:astro-ph/0009005].

[42] R. Bottema, "The maximum rotation of a galactic disc", astro-ph/9706230.

[43] E. F. Bell and R. S. de Jong, Astrophys. J. 550, 212 (2001) arXiv:astro-ph/0011493.

[44] A. Portas, E. Brinks, A. Usero, F. Walter, W. J. G. de Blok, Jr., R. C. Kennicutt, in The Galaxy Disk in Cosmological Context, Proceedings of IAU Symposium No. 254, eds. J. Andersen, J. Bland-Hawthorn \& B. Nordström (Cambridge: Cambridge University Press), p 52 


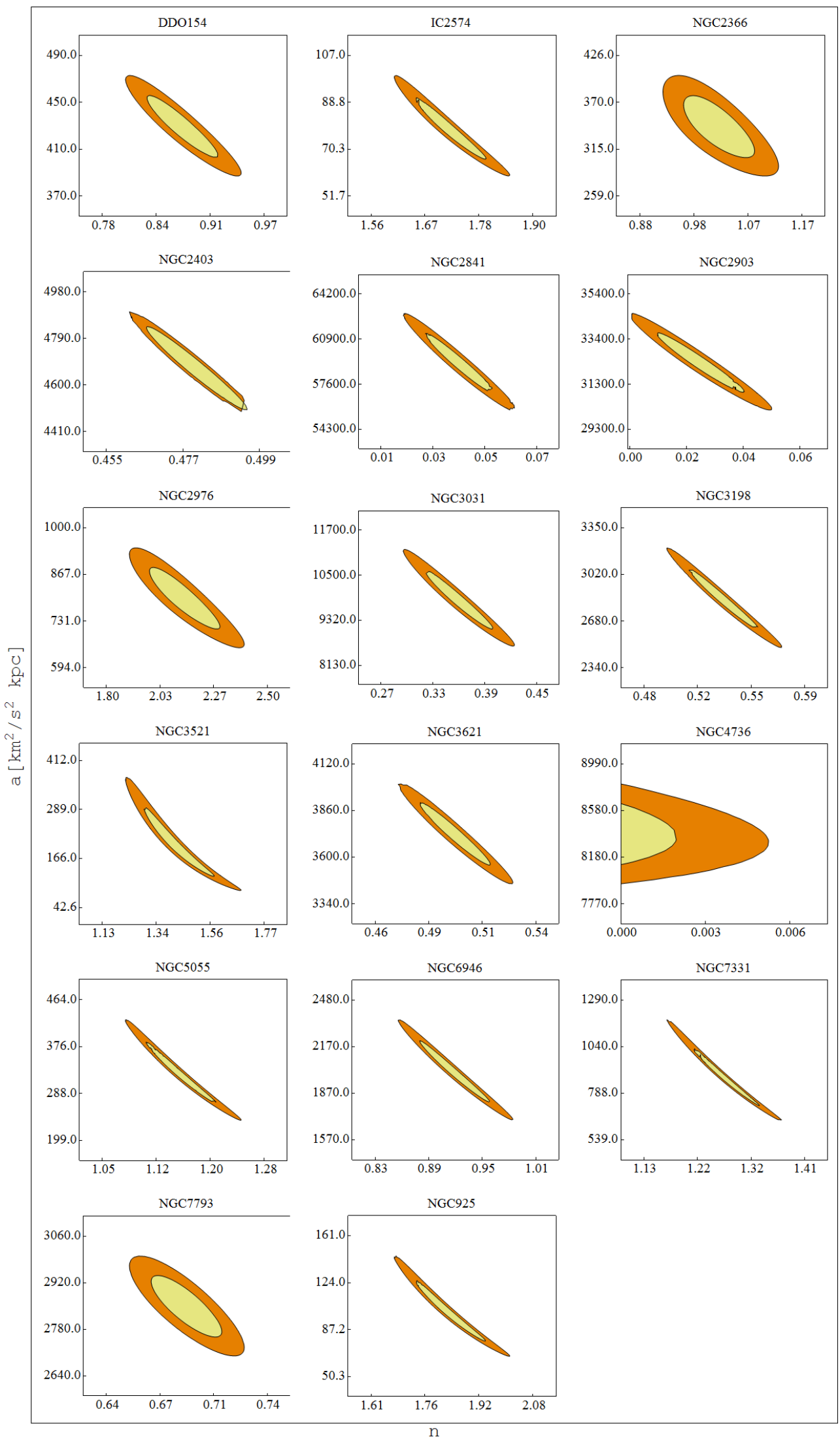

FIG. 1: 2D likelihood contours between the $a$ and $n$, corresponding to $1 \sigma$ and $2 \sigma$, for the Kroupa IMF model. 


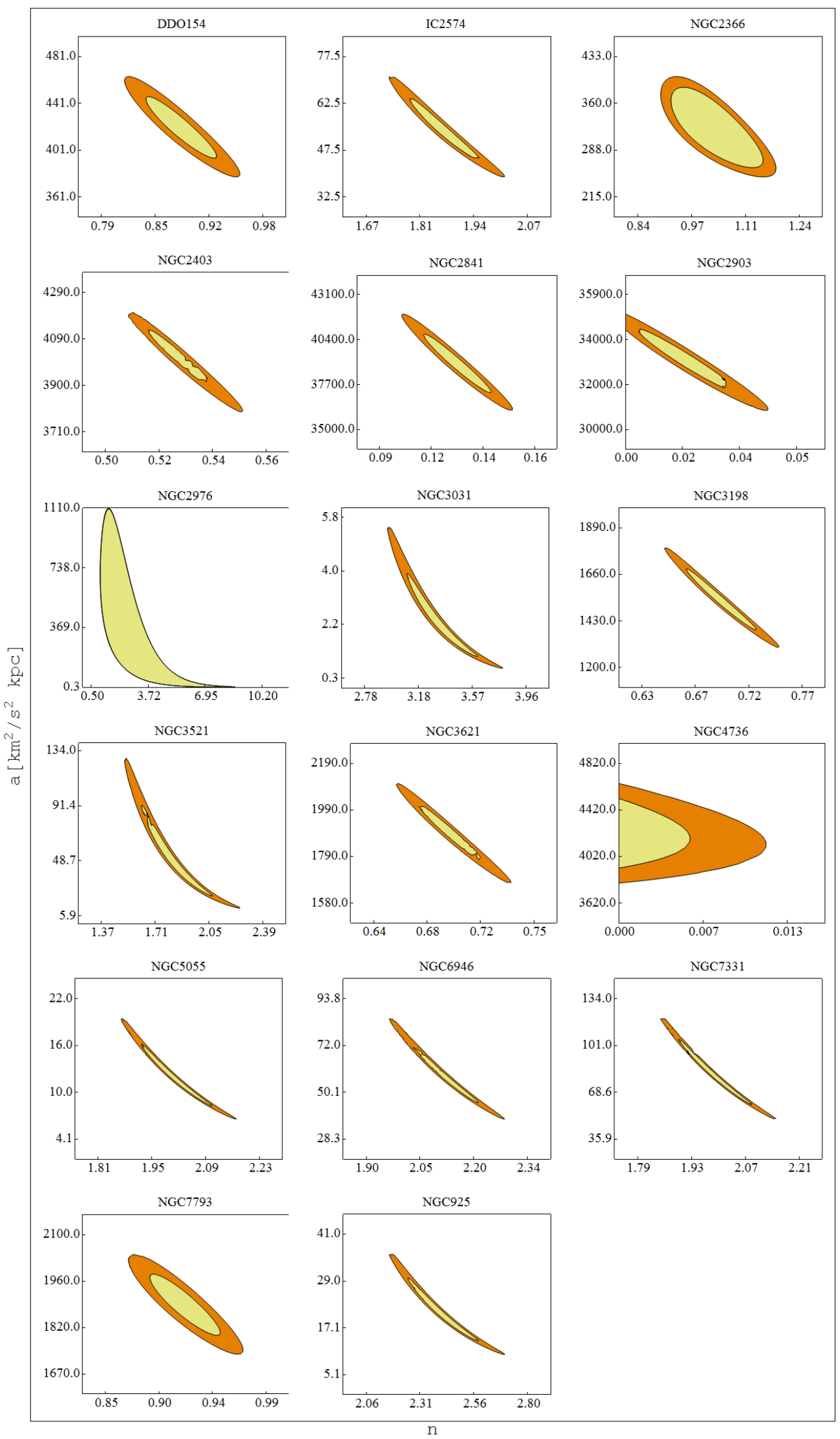

FIG. 2: 2D likelihood contours between the $a$ and $n$, corresponding to $1 \sigma$ and $2 \sigma$, for the diet-Salpeter IMF model. 


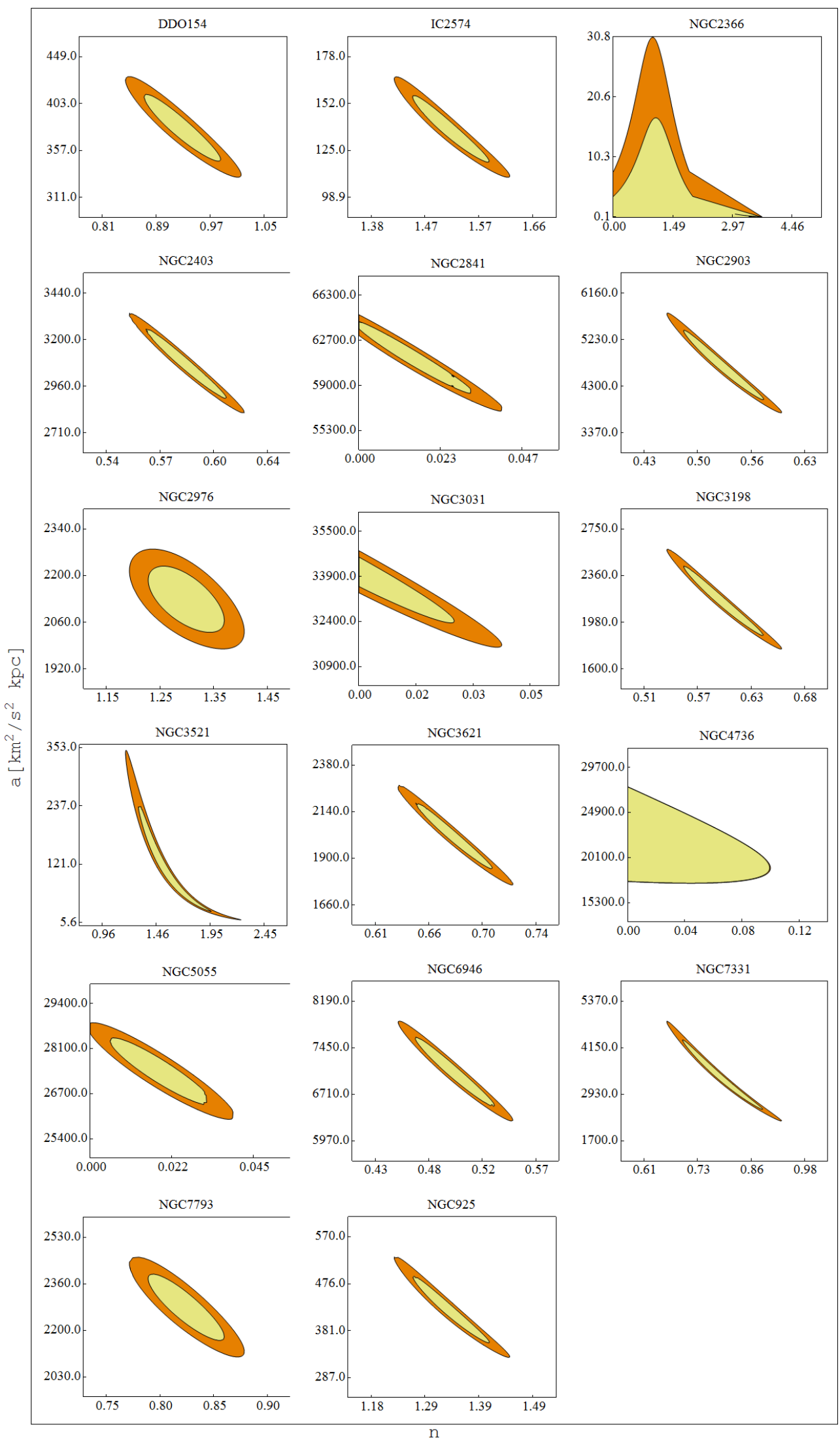

FIG. 3: 2D likelihood contours between the $a$ and $n$, corresponding to $1 \sigma$ and $2 \sigma$, for the free mass model. 


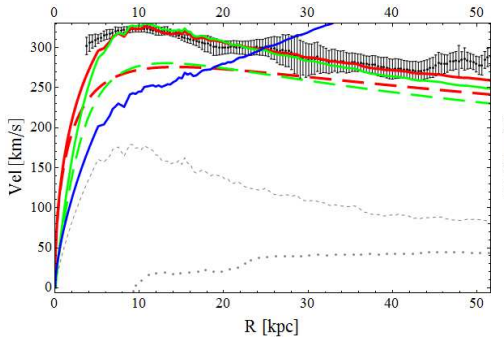

(a) N 2841

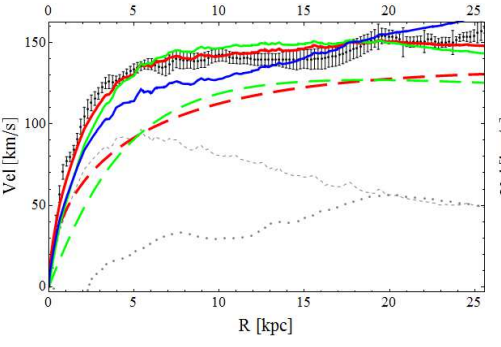

(b) N 3621

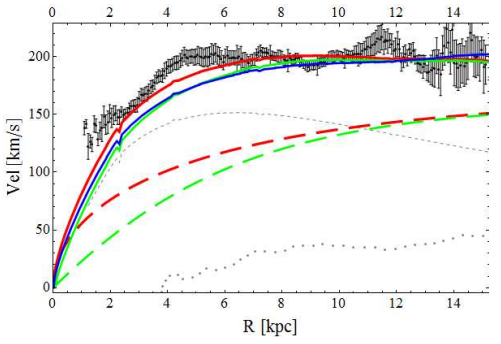

(c) N 6946

FIG. 4: Rotation curve for the galaxies (a) N 2841, (b) N 3621, and (c) N 6946. We explicitly plot the contribution of the gas (dotted, black), the Kroupa star model (dashed, black), the red/green plots are the NFW/Burkert DM halo profile and its total contribution (dashed and thick lines, respectively), and finally the blue line is the total rotation curve of the standard $(n=1)$ Rindler acceleration model. 